\title{
Modulatory effect of coffee fruit extract on plasma levels of brain-derived neurotrophic factor in healthy subjects
}

\author{
Tania Reyes-Izquierdo ${ }^{1 *}$, Boris Nemzer ${ }^{2}$, Cynthia Shu ${ }^{1}$, Lan Huynh ${ }^{1}$, Ruby Argumedo ${ }^{1}$, Robert Keller ${ }^{3}$ \\ and $\mathrm{Zb}$ Pietrzkowski ${ }^{1}$ \\ ${ }^{1}$ Applied BioClinical, Inc., 16259 Laguna Canyon Road, Irvine, CA 92618, USA \\ ${ }^{2}$ FutureCeuticals, Inc., 2692 North State Route 1-17, Momence, IL 60954, USA \\ ${ }^{3}$ NutraClinical, Inc., 5755 Oberlin Drive, Suite 301, San Diego, CA 92121, USA
}

(Submitted 6 July 2012 - Final revision received 30 October 2012 - Accepted 30 October 2012 - First published online 14 January 2013$)$

\begin{abstract}
The present single-dose study was performed to assess the effect of whole coffee fruit concentrate powder (WCFC), green coffee caffeine powder (N677), grape seed extract powder (N31) and green coffee bean extract powder (N625) on blood levels of brain-derived neurotrophic factor (BDNF). Randomly assorted groups of fasted subjects consumed a single, $100 \mathrm{mg}$ dose of each material. Plasma samples were collected at time zero $\left(T_{0}\right)$ and at $30 \mathrm{~min}$ intervals afterwards, up to $120 \mathrm{~min}$. A total of two control groups were included: subjects treated with silica dioxide (as placebo) or with no treatment. The collected data revealed that treatments with N31 and N677 increased levels of plasma BDNF by about $31 \%$ under these experimental conditions, whereas treatment with WCFC increased it by $143 \%$ ( $n$ 10), compared with baseline. These results indicate that WCFC could be used for modulation of BDNF-dependent health conditions. However, larger clinical studies are needed to support this possibility.
\end{abstract}

Key words: Brain-derived growth factor: Coffee fruit extract: Caffeine: Polyphenols: Procyanidins

Brain-derived neurotrophic factor (BDNF) is a member of the nerve growth factor-related family ${ }^{(1)}$ and is a homodimeric protein that has been highly conserved in structure and function during evolution ${ }^{(2,3)}$. BDNF is a secreted protein that, in human subjects, is encoded by the $B D N F$ gene ${ }^{(3)}$. It is involved in development, maintenance and function of the central nervous system $^{(4,5)}$. BDNF is found in a wide range of tissues ${ }^{(3)}$ and it can be self-regulated ${ }^{(6,7)}$. BDNF has several documented shortand long-term functional roles. It is now well-known that BDNF serves as a target-derived survival and differentiation factor for neuronal sub-populations in prenatal stages ${ }^{(8-10)}$. Likewise, BDNF promotes long-term potentiation by potentiating excitatory neurotransmitter activity in the hippocampus ${ }^{(11)}$ and $\alpha$-amino-3-hydroxy-5-methyl-4-isoxazolepropionic acid (AMPA) receptor insertion post-synaptically ${ }^{(12)}$. These actions, along with BDNF's ability to stabilise dendritic spines ${ }^{(13)}$, underlie the molecule's important role in learning, memory and behaviour ${ }^{(7)}$. In addition, BDNF levels increase in response to certain forms of injury, such as ischaemic-hypoxic ${ }^{(14)}$ and infectious insults ${ }^{(15)}$, presumably by blocking apoptosis. Various studies have shown a link between BDNF and certain health conditions, such as depression ${ }^{(16,17)}$, obsessive-compulsive disorder ${ }^{(18)}$, Alzheimer's disease $^{(19)}$, dementia ${ }^{(20)}$ and Parkinson's disease ${ }^{(21,22)}$.

Despite its name, BDNF is found in a variety of tissues and cell types, not just in the brain. It is also expressed in cardiovascular, immune, reproductive and endocrine tissues ${ }^{(4,5,23)}$. Intact BDNF readily crosses the blood-brain barrier via a high-capacity transporter system ${ }^{(24)}$. Interestingly, exercise has been shown to increase the expression of BDNF in human subjects ${ }^{(25-27)}$ and a similar effect was observed after caffeine application in vitro ${ }^{(28)}$ and in vivo ${ }^{(29)}$. Caffeine has been recently proposed as a potential candidate for maintaining physiological levels of BDNF, as it is capable of positively affecting cognition ${ }^{(29,30)}$. In the present study, we tested polyphenol-rich natural products containing different amount of caffeine to see if a single dose could lead to an increase in plasma levels of BDNF. Three natural products containing varying amounts of caffeine were selected for the present pilot study: green coffee caffeine (N677), green coffee bean extract (N625) and whole coffee fruit concentrate powder $(\mathrm{WCFC})^{(31)}$. A grape seed extract (N31) containing high levels of polyphenols, but not caffeine ${ }^{(32)}$, was also tested.

Abbreviations: BDNF, brain-derived neurotrophic factor; N31, grape seed extract powder; N625, green coffee bean extract powder; N677, green coffee caffeine powder; WCFC, whole coffee fruit concentrate powder.

*Corresponding author: T. Reyes-Izquierdo, fax +1949 502 4987, email tania@abclinicaldiscovery.com 


\section{Materials and methods}

\section{Materials}

Extract powders tested in the present study were provided by FutureCeuticals, Inc. WCFC is a patented extract of whole coffee fruit (coffee berries) from Coffea arabica. Chemical composition and polyphenol profiles of each tested extract appear in Table 1. Dulbecco's PBS and water were purchased from Sigma Chemical Company. Protein low binding microtubes were obtained from Eppendorf and RC DC Protein Assay Kit II was purchased from Bio-Rad. Human BDNF Quantikine ELISA kits were from R\&D Systems. Heparin blood collection tubes were obtained from Ram Scientific, Inc. and lancets were purchased from Medlance ${ }^{\circledR}$. Silica oxide used as placebo was also purchased from Sigma Chemical Company.

\section{Study description}

The present study was conducted according to the guidelines laid down in the Declaration of Helsinki, and all procedures involving human subjects/patients were approved by the Institutional Review Board at Vita Clinical S.A. Avenida Circunvalacion Norte \#135, Guadalajara, JAL, Mexico 44270 (study protocol no. 12-06 ABC-BDNF). The present pilot acute clinical study was performed on healthy fasted subjects treated

Table 1. Amount of caffeine, polyphenols and procyanidins in extracts tested in the present study

\begin{tabular}{|c|c|c|c|c|}
\hline \multirow[b]{2}{*}{ Compound } & \multicolumn{4}{|c|}{$\begin{array}{l}\text { Chemical fingerprint of tested } \\
\text { extracts }\end{array}$} \\
\hline & WCFC & N677 & N625 & N31 \\
\hline Caffeine (\%) & 0.7 & $72 \cdot 8$ & 2 & ND \\
\hline Total polyphenols (\%) & $47 \cdot 8$ & $2 \cdot 8$ & 40 & 72 \\
\hline Total chlorogenic acids (\%) & $46 \cdot 6$ & $2 \cdot 1$ & 40 & None \\
\hline 5-O-Caffeoylquinic acid (\%) & $20 \cdot 4$ & 0.9 & $16 \cdot 8$ & None \\
\hline 3-O-Caffeoylquinic acid (\%) & $5 \cdot 5$ & 0.2 & $5 \cdot 1$ & None \\
\hline 4-O-Caffeoylquinic acid (\%) & $7 \cdot 8$ & 0.4 & 7.9 & None \\
\hline 4-O-Feruloylquinic acid (\%) & 0.7 & $<0.1$ & 0.8 & None \\
\hline 5-O-Feruloylquinic acid (\%) & 2.5 & 0.1 & $2 \cdot 3$ & None \\
\hline 3,4-O-Dicaffeoylquinic acid (\%) & $2 \cdot 8$ & 0.1 & $2 \cdot 7$ & None \\
\hline 3,5-O-Dicaffeoylquinic acid (\%) & $2 \cdot 3$ & 0.1 & $2 \cdot 4$ & None \\
\hline 4,5-O-Dicaffeoylquinic acid (\%) & $3 \cdot 7$ & 0.2 & 3.0 & None \\
\hline $\begin{array}{l}\text { 4-O-Caffeoyl-5-O-feoylquinic } \\
\text { acid (\%) }\end{array}$ & 0.3 & $<0.1$ & 1.0 & None \\
\hline Trigonelline (\%) & $3 \cdot 2$ & $<0.1$ & $2 \cdot 7$ & None \\
\hline Procyanidin dimmer $B_{2}(\mu \mathrm{g} / \mathrm{g})$ & $27 \cdot 2$ & None & $<0.1$ & $5 \cdot 6$ \\
\hline Quercetin-3-rutinoside $(\mu \mathrm{g} / \mathrm{g})$ & 23.5 & None & $<0.1$ & None \\
\hline Procyanidin trimer $B_{2}(\mu \mathrm{g} / \mathrm{g})$ & $16 \cdot 3$ & None & $<0.1$ & $1 \cdot 8$ \\
\hline Procyanidin tetramer $B_{2}(\mu \mathrm{g} / \mathrm{g})$ & 4.4 & None & $<0.1$ & $1 \cdot 7$ \\
\hline$+(-)$-Catechin $(\mu \mathrm{g} / \mathrm{g})$ & $1 \cdot 1$ & None & $<0.1$ & 4.5 \\
\hline$-(-)$-Epicatechin $(\mu \mathrm{g} / \mathrm{g})$ & 17.9 & None & $<0.1$ & $7 \cdot 1$ \\
\hline ORAC $(T E / \mu g)$ & 8445 & 245 & 3050 & 15656 \\
\hline HORAC $(T E / \mu \mathrm{g})$ & 21920 & 515 & 7098 & 34220 \\
\hline SORAC $(T E / \mu \mathrm{g})$ & 860 & 7 & 282 & 23895 \\
\hline NORAC $(\mathrm{TE} / \mu \mathrm{g})$ & 527 & 28 & 168 & 657 \\
\hline $\operatorname{SOAC}(\mathrm{TE} / \mu \mathrm{g})$ & 2042 & 89 & 686 & 3928 \\
\hline
\end{tabular}

WCFC, whole coffee fruit concentrate powder; N677, green coffee caffeine powder; N625, green coffee bean extract powder; N31, grape seed extract powder; ND, not determined; ORAC, oxygen radical absorbance capacity; TE, Trolox ${ }^{\mathrm{TM}}$ equivalents; HORAC, hydroxyl oxygen radical absorbance capacity; SORAC, superoxide radical absorbance capacity; NORAC, peroxynitrite radical absorbance capacity; SOAC, singlet oxygen radical absorbance capacity. once with a single dose of tested material, placebo (silica oxide) or vehicle only (water). All study subjects were generally healthy, non-smokers and did not use any type of medication or supplement for a period of $15 \mathrm{~d}$ prior to the start of the study. The inclusion criteria required participants to be between the ages of 18 and 55 years and have a BMI between 18.0 and $25.0 \mathrm{~kg} / \mathrm{m}^{2(33)}$. At the time of the study, participants were free of rhinitis, influenza and other symptoms of upper respiratory infection. To minimise confounding effects, all subjects remained in the testing facility during experimentation to avoid the possibility of blood BDNF increase due to physical activity and exercise ${ }^{(34)}$. In addition, all subjects were tested during the same time of day to minimise any differences in blood BDNF due to diurnal effect ${ }^{(35)}$. Participants were excluded if they had diabetes mellitus, a known allergy to any of the test ingredients or were using any antiinflammatory, analgesic, anti-allergy, anti-depressant medication or multivitamins. Participants received oral and written information about the experimental procedures before giving their written consent.

For the present study, twenty-five ${ }^{(25)}$ subjects were randomly divided into groups of five to receive one of the five treatments: N677, N625, N31, WCFC or placebo (silica oxide). In follow-up studies performed under the same experimental conditions, five participants received WCFC, chlorogenic acid or water as vehicle (no treatment group). In all cases, subjects fasted for $12 \mathrm{~h}$ prior to the first blood collection. Other than consuming one of the study materials or placebo, patients had no intake per os during the study period. Blood was collected at baseline $\left(T_{0}\right)$ and subsequent samples were collected at every $30 \mathrm{~min}\left(T_{30}, T_{60}, T_{90}\right.$ and $\left.T_{120}\right)$ after the treatment.

\section{Brain-derived neurotrophic factor detection and quantification}

For the isolation of plasma, $100 \mu \mathrm{l}$ finger blood were collected by finger puncture and placed in Safe-T-Fill ${ }^{\circledR}$ Capillary blood collection tubes (Ram Scientific, Inc.) and centrifuged at $1000 \mathrm{~g}$ for $10 \mathrm{~min}$. Blood was transferred to protein low binding tubes and kept at $-80^{\circ} \mathrm{C}$ until use. BDNF was measured using a quantitative sandwich ELISA immunoassay (R\&D Systems) following the instructions provided in the kit, using the buffers and calibrators specific for plasma. Final reactions were measured using a spectrophotometer (Molecular Devices) at 450 and $540 \mathrm{~nm}$ wavelengths, and final concentrations were calculated from a standard curve.

\section{Chemical analyses}

Chlorogenic acid, procyanidins, flavanols and flavonols of WCFC, N625 and N31 were characterised by LC-MS( $n$ ) and quantified by UV absorbance ${ }^{(36)}$. Total polyphenol content was determined by spectrophotometry according to the Folin-Ciocalteu method ${ }^{(37)}$, and was calibrated against gallic acid standard (Sigma-Aldrich). Results were expressed as grams of gallic acid equivalents. 
The caffeine and trigonelline contents were characterised by HPLC Agilent 1100 (Agilent Technologies) equipped with a diode array detector and quantified by UV absorbance (W Mullen, B Nemzer, M Clifford, et al., unpublished results). Antioxidant capacities of coffee fruit extract, coffee bean extract and N31 were characterised by the ability of the samples to scavenge peroxyl radicals (oxygen radical absorbance capacity), hydroxyl radicals (hydroxyl oxygen radical absorbance capacity), peroxynitrite (peroxynitrite radical absorbance capacity), superoxide anions (superoxide radical absorbance capacity) and singlet oxygen (singlet oxygen radical absorbance capacity) ${ }^{(39-41)}$.

\section{Statistical analysis}

BDNF levels were compared with a reference standard curve and each subject was normalised to their own value measured at time zero $\left(T_{0}\right)$. Peak levels of plasma BDNF for each patient were used for comparisons. Results were pooled and standard error of the mean was used for each separate analysis. Plasma BDNF levels at 60 min after treatment were compared with baseline using a two-tail, independent Student's $t$ test. Power analyses were run using G*Power Data Analysis (HeinrichHeine-University Düsseldorf). Statistical power was run for the whole group and it was also calculated per-pair power for individual comparison. Descriptive analysis was run in GraphPad to derive the mean and standard deviation for each group.

\section{Results and discussion}

Four polyphenol-rich fruit extracts were tested in healthy subjects. Three of the extracts contained caffeine in varying amounts (WCFC: $0.7 \%$ caffeine; N677: 72.8\% caffeine; and N625: 2\% caffeine) and one extract was caffeine free (N31) (see Table 1). Of the substances tested, WCFC increased BDNF plasma levels (Fig. 1) in patients by an average of $137 \%$ with respect to baseline (range $65-222 \% ; P=0.001$ $v$. placebo). N677 showed an increase of $42 \%$, but was not statistically significant $(P=0 \cdot 49)$. N625 did not cause a significant increase in BDNF. N31 increased BDNF levels in

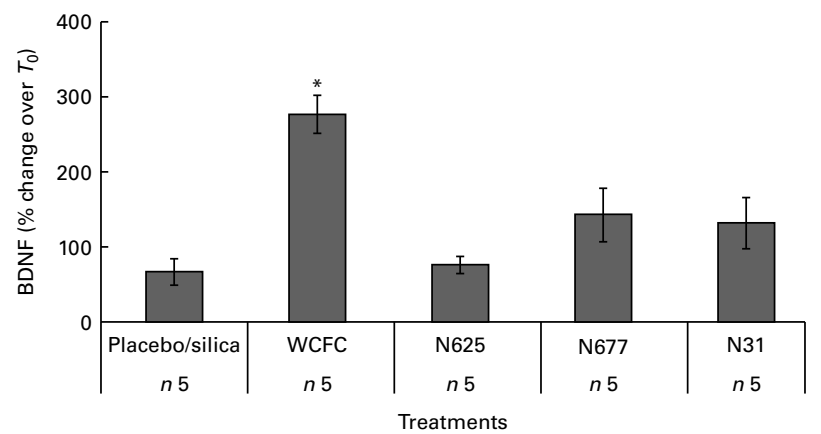

Fig. 1. Blood levels of brain-derived neurotrophic factor (BDNF) collected from subjects treated with whole coffee fruit concentrate powder (WCFC), green coffee bean extract powder (N625), green coffee caffeine powder (N677), grape seed extract powder (N31) and placebo (silica). Data represent average percentage difference from administration $\left(T_{0}\right)$. * Mean value was significantly different compared with placebo by Student's $t$ test $(P<0.05)$.

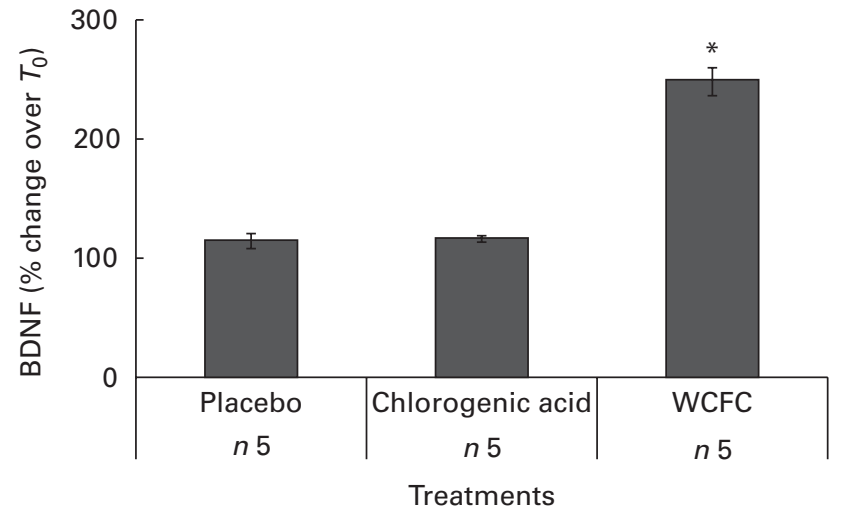

Fig. 2. Blood levels of brain-derived neurotrophic factor (BDNF) measured in non-treated subjects (placebo), subjects receiving $50 \mathrm{mg}$ of chlorogenic acid or $100 \mathrm{mg}$ whole coffee fruit concentrate powder (WCFC). Data are presented as the average percentage difference compared with baseline $\left(T_{0}\right)$. * Mean value was significantly different compared with placebo by Student's $t$ test $(P<0.05)$

plasma by $30 \%$ with respect to baseline, though not significantly $(P=0.65)$.

It is important to note that a power analysis of the entire sample indicated that forty subjects would be required to reach a power of $80 \%$. Our sample size was twenty-five, therefore one must interpret the lack of BDNF effect of N677, N625 and N31 with caution. Larger groups are required to make definitive conclusions for these treatments. However, the result of this power analysis does not diminish the statistical significance of WCFC on plasma BDNF levels. Indeed, a separate per-pair power analysis of WCFC and placebo showed a sample size of ten to have a greater than $99 \%$ power.

Quite unexpectedly, treatment with placebo (silica dioxide) resulted in a $34 \%$ reduction in BDNF blood levels $(P=0 \cdot 09)$. Silica oxide was used as placebo, as it is generally considered to be an inert material. It has been previously reported that stress can decrease plasma levels of BDNF in some human and animal models ${ }^{(42-44)}$. However, the reduction of plasma BDNF due to the intake of silica/placebo has not been reported. Consequently, a second experiment was performed to verify the reproducibility of the effect of WCFC on BDNF and to test the effect of extended fasting (untreated control) on the baseline level of BDNF in blood.

A total of five additional healthy subjects who met the same inclusion criteria were treated with $100 \mathrm{mg}$ WCFC. As before, all groups fasted for $12 \mathrm{~h}$ prior to testing, but the control group did not receive any treatment besides water as vehicle $(200 \mathrm{ml})$ as it was used for treatment with WCFC. As shown in Fig. 2, BDNF plasma levels were increased in subjects treated with $100 \mathrm{mg}$ of WCFC ( $148 \%$ increase; $P=0 \cdot 002)$. However, blood BDNF level in the untreated group was not statistically changed ( $15 \%$ increase by average).

Pooling all ten subjects from the two studies, treatment with $100 \mathrm{mg}$ of WCFC caused a 143\% increase in BDNF plasma levels. The stimulatory effect of caffeine on BDNF has been previously observed ${ }^{(29,30,45,46)}$. However, as presented in Fig. 1, single-dose treatment with caffeine-containing extracts 
resulted in an increased level of plasma BDNF in a caffeine concentration-independent manner. As shown in Table 1, N677 is mostly comprised of caffeine ( $72.8 \%$ by weight), yet caused only modest increases in plasma BDNF levels. The most profound increases in plasma BDNF were observed after treatment with WCFC, although this extract contains only $0.7 \%$ caffeine by weight.

Previous reports have shown that procyanidins extracted from grape seeds are capable of stimulating neurotrophic factors in aged rats ${ }^{(47)}$. These compounds have also been implicated in the regulation of metabolic disorders ${ }^{(48,49)}$ that are BDNF dependent. The N31 used in the present study had relatively high polyphenol levels compared with the coffee fruit, coffee caffeine and coffee seed extracts, yet failed to significantly increase BDNF in blood.

This result suggests that the stimulatory effect of WCFC on the blood level of BDNF is not associated with the amount of polyphenols or caffeine per dose. Rather, the effect may be related to either the amount of procyanidins or to the unique coffee polyphenol profile of the WCFC material. According to the present analyses, WCFC shows a significant amount of procyanidins in comparison to N31, N625 and N677 (Table 1), suggesting that acute treatment with procyanidin-rich whole coffee fruit extracts (and possibly other procyanidin-rich extracts), may increase blood levels of BDNF in human subjects. Future work could include testing other procyanidin-rich extracts for their ability to raise plasma BDNF in order to confirm this hypothesis.

The amount of trigonelline also varied in each material tested (Table 1). As presented, WCFC and N625 contain the highest amount of this compound; however, the effect of WCFC on plasma BDNF is superior to the effect of N625 under the same experimental conditions, suggesting that this is not the primary agent responsible for increased BDNF levels in blood.

As WCFC contains high amounts of chlorogenic acid, it was hypothesised that this specific polyphenolic acid may cause an increase in blood level of BDNF. Consequently, we administered $50 \mathrm{mg}$ of chlorogenic acid as a single dose to five healthy subjects. As presented in Fig. 2, chlorogenic acid did not increase blood level of BDNF in a statistically significant manner $(P=0 \cdot 89)$, suggesting that this substance is not responsible for the ability of WCFC to increase BDNF.

The compounds tested in the present study had different concentrations of caffeine and polyphenols (Table 1). It is important to note that the present results show the percentage of caffeine within each tested compound, rather than an absolute mass of caffeine. Nevertheless, the present work suggests that procyanidins may have the ability to increase plasma BDNF levels and, perhaps, to a larger extent than caffeine itself. This is particularly interesting considering that recent research show that procyanidin oligomers play a role in neuroprotection from excitotoxic injury ${ }^{(50)}$.

BDNF-dependent telomerase activity has been shown to promote neuron survival in developing hippocampal neurons ${ }^{(51)}$. Increased BDNF expression and telomerase activity after brain injury suggest that telomerase may play a role in BDNF-mediated neuroprotection. Furthermore, BDNF has been shown to up-regulate telomerase expression and activity in spinal motor neurons ${ }^{(52)}$. These neurons, treated with BDNF, are more resistant to ex-cytotoxic injury, presumably from increased cellular resistance to apoptosis. It is interesting to speculate that WCFC possibly may also exert an antiapoptotic effect through telomerase by increased BDNF activity.

WCFC was tested under experimental conditions reducing impact of possible confounding effects; for example, nonsmokers were not included in the present study, since nicotine is known to increase blood levels of $\mathrm{BDNF}^{(53)}$. All subjects remained in the clinical site during the duration of the study to avoid any shifts in blood level of BDNF due to exercise and physical activity ${ }^{(34)}$. Finally, the present clinical study was performed in the morning starting at 08.00 hours, as it was reported that blood levels of BDNF are increased due to diurnal effect ${ }^{(35)}$.

In summary, WCFC increased the blood level of BDNF during first $60 \mathrm{~min}$ after treatment with a dose of $100 \mathrm{mg}$. In order to confirm the results of the present pilot study, further clinical testing in a larger group is required.

\section{Acknowledgements}

The present study was funded by Futureceuticals, Inc. T. R.-I. conducted the experimental work, analysed the data and led the manuscript writing. C. S., L. H. and R. A. performed serum tests and helped in the data analysis. R. K. organised and helped execute clinical protocol of the study. B. N. designed and conducted all the chemical analysis. Z. P. designed and directed the study. We would like to thank Michael Sapko for his help in editing the manuscript. All authors declare that they have no conflicts of interest.

\section{References}

1. Fujinami A, Ohta K, Obayashi H, et al. (2008) Serum brainderived neurotrophic factor in patients with type 2 diabetes mellitus: relationship to glucose metabolism and biomarkers of insulin resistance. Clin Biochem 41, 812-817.

2. Jones KR \& Reichardt LF (1990) Molecular cloning of a human gene that is a member of the nerve growth factor family. Proc Natl Acad Sci U S A 87, 8060-8064.

3. Maisonpierre PC, Le Beau MM, Espinosa R III, et al. (1991) Human and rat brain-derived neurotrophic factor and neurotrophin-3: gene structures, distributions, and chromosomal localizations. Genomics 10, 558-568.

4. Tessarollo L (1998) Pleiotropic functions of neurotrophins in development. Cytokine Growth Factor Rev 9, 125-137.

5. Yamamoto M, Sobue G, Yamamoto K, et al. (1996) Expression of mRNAs for neurotrophic factors (NGF, BDNF, NT-3, and GDNF) and their receptors (p75NGFR, trkA, trkB, and trkC) in the adult human peripheral nervous system and nonneural tissues. Neurochem Res 21, 929-938.

6. Cunha C, Angelucci A, D'Antoni A, et al. (2009) Brainderived neurotrophic factor (BDNF) overexpression in the forebrain results in learning and memory impairments. Neurobiol Dis 33, 358-368.

7. Cunha C, Brambilla R \& Thomas KL (2010) A simple role for BDNF in learning and memory? Front Mol Neurosci 3, 1. 
8. Grothe C \& Unsicker K (1987) Neuron-enriched cultures of adult rat dorsal root ganglia: establishment, characterization, survival, and neuropeptide expression in response to trophic factors. J Neurosci Res 18, 539-550.

9. Kalcheim C \& Gendreau M (1988) Brain-derived neurotrophic factor stimulates survival and neuronal differentiation in cultured avian neural crest. Brain Res 469, 79-86.

10. Snider WD (1994) Functions of the neurotrophins during nervous system development: what the knockouts are teaching us. Cell 77, 627-638.

11. Boulanger LM \& Poo MM (1999) Presynaptic depolarization facilitates neurotrophin-induced synaptic potentiation. Nat Neurosci 2, 346-351.

12. Li W \& Keifer J (2008) Coordinate action of pre- and postsynaptic brain-derived neurotrophic factor is required for AMPAR trafficking and acquisition of in vitro classical conditioning. Neuroscience 155, 686-697.

13. Tanaka J, Horiike Y, Matsuzaki M, et al. (2008) Protein synthesis and neurotrophin-dependent structural plasticity of single dendritic spines. Science (New York, NY) 319, $1683-1687$.

14. Han BH \& Holtzman DM (2000) BDNF protects the neonatal brain from hypoxic-ischemic injury in vivo via the ERK pathway. J Neurosci 20, 5775-5781.

15. Bifrare Y-D, Kummer J, Joss P, et al. (2005) Brain-derived neurotrophic factor protects against multiple forms of brain injury in bacterial meningitis. J Infect Dis 191, 40-45.

16. Brunoni AR, Lopes $M \&$ Fregni $F$ (2008) A systematic review and meta-analysis of clinical studies on major depression and BDNF levels: implications for the role of neuroplasticity in depression. Int J Neuropsychopharmacol 11, 1169-1180.

17. Dwivedi Y (2009) Brain-derived neurotrophic factor: role in depression and suicide. Neuropsychiatr Dis Treat $\mathbf{5}$, 433-449.

18. Maina G, Rosso G, Zanardini R, et al. (2010) Serum levels of brain-derived neurotrophic factor in drug-naive obsessivecompulsive patients: a case-control study. I Affect Disord 122, $174-178$

19. Zuccato C \& Cattaneo E (2009) Brain-derived neurotrophic factor in neurodegenerative diseases. Nat Rev Neurol 5 , 311-322.

20. Arancio O \& Chao MV (2007) Neurotrophins, synaptic plasticity and dementia. Curr Opin Neurobiol 17, 325-330.

21. Fumagalli F, Racagni G \& Riva MA (2006) Shedding light into the role of BDNF in the pharmacotherapy of Parkinson's disease. Pharmacogenomics J 6, 95-104.

22. Chun HS, Son JJ \& Son JH (2000) Identification of potential compounds promoting BDNF production in nigral dopaminergic neurons: clinical implication in Parkinson's disease. Neuroreport 11, 511-514.

23. Seifer DB, Feng B, Shelden RM, et al. (2002) Brain-derived neurotrophic factor: a novel human ovarian follicular protein. J Clin Endocrinol Metab 87, 655-659.

24. Pan W, Banks WA, Fasold MB, et al. (1998) Transport of brain-derived neurotrophic factor across the blood-brain barrier. Neuropharmacology 37, 1553-1561.

25. Kramer AF, Colcombe SJ, McAuley E, et al. (2005) Fitness, aging and neurocognitive function. Neurobiol Aging 26, Suppl. 1, 124-127.

26. Colcombe SJ, Kramer AF, McAuley E, et al. (2004) Neurocognitive aging and cardiovascular fitness: recent findings and future directions. J Mol Neurosci 24, 9-14.

27. Kramer AF, Hahn S, Cohen NJ, et al. (1999) Ageing, fitness and neurocognitive function. Nature 400, 418-419.
28. Connolly SK \& Tamy (2010) Caffeine modulates CREBdependent gene expression in developing cortical neurons. Biochem Biophys Res Commun 397, 152-156.

29. Costa MS, Botton PH, Mioranzza S, et al. (2008) Caffeine improves adult mice performance in the object recognition task and increases BDNF and TrkB independent on phospho-CREB immunocontent in the hippocampus. Neurochem Int 53, 89-94.

30. Alhaider IA, Aleisa AM, Tran TT, et al. (2011) Sleep deprivation prevents stimulation-induced increases of levels of P-CREB and BDNF: protection by caffeine. Mol Cell Neurosci 46, 742-751.

31. Heimbach JT, Marone PA, Hunter JM, et al. (2010) Safety studies on products from whole coffee fruit. Food Chem Toxicol 48, 2517-2525.

32. Yashin YI, Nemzer BV, Ryzhnev VY, et al. (2010) Creation of a databank for content of antioxidants in food products by an amperometric method. Molecules 15, 7450-7466.

33. Borecki IB, Higgins M, Schreiner PJ, et al. (1998) Evidence for multiple determinants of the body mass index: the National Heart, Lung, and Blood Institute Family Heart Study. Obes Res 6, 107-114.

34. Zolodz JAPA, Majerczak K, Grandys M, et al. (2008) Endurance training increases brain-derived neurotrophic factor concentration in young healthy men. J Physiol Pharmacol S17, 59, 199-132.

35. Piccini A, Marazziti D, Del Debbio A, et al. (2008) Diurnal variation of plasma brain-derived neurotrophic growth factor (BDNF) in humans. Chronobiol Int 25, 819-826.

36. Mullen W, Nemzer B, Ou B, et al. (2011) The antioxidant and chlorogenic acid profiles of whole coffee fruits are influenced by the extraction procedures. J Agric Food Chem 59, $3754-3762$.

37. International Organization for Standardization (ISO) (2005) Determination of Substances Characteristic of Green and Black Tea. Part 1: Content of Total Polyphenols in Tea Colorimetric Method Using Folin-Ciocalteu Reagent. Geneva: ISO. ISO 14502-1.

38. Chung HY, Choi HR, Park HJ, et al. (2001) Peroxynitrite scavenging and cytoprotective activity of 2,3,6-tribromo4,5-dihydroxybenzyl methyl ether from the marine alga Symphyocladia latiuscula. J Agric Food Chem 49, 3614-3621.

39. Huang D, Ou B, Hampsch-Woodill M, et al. (2002) Highthroughput assay of oxygen radical absorbance capacity (ORAC) using a multichannel liquid handling system coupled with a microplate fluorescence reader in 96-well format. J Agric Food Chem 50, 4437-4444.

40. Ou B, Hampsch-Woodill M, Flanagan J, et al. (2002) Novel fluorometric assay for hydroxyl radical prevention capacity using fluorescein as the probe. J Agric Food Chem 50, 2772-2777.

41. Zhang L, Huang D, Kondo M, et al. (2009) Novel highthroughput assay for antioxidant capacity against superoxide anion. J Agric Food Chem 57, 2661-2667.

42. Elzinga BM, Molendijk ML, Oude Voshaar RC, et al. (2011) The impact of childhood abuse and recent stress on serum brain-derived neurotrophic factor and the moderating role of BDNF Val66Met. Psychopharmacology 214, 319-328.

43. Larsen MH, Mikkelsen JD, Hay-Schmidt A, et al. (2010) Regulation of brain-derived neurotrophic factor (BDNF) in the chronic unpredictable stress rat model and the effects of chronic antidepressant treatment.JPsychiatr Res 44, 808-816.

44. Advani T, Koek W \& Hensler JG (2009) Gender differences in the enhanced vulnerability of BDNF $+/-$ mice to mild stress. Int J Neuropsychopharmacol 12, 583-588.

45. Capiotti KM, Menezes FP, Nazario LR, et al. (2011) Early exposure to caffeine affects gene expression of adenosine 
receptors, DARPP-32 and BDNF without affecting sensibility and morphology of developing zebrafish (Danio rerio). Neurotoxicol Teratol 33, 680-685.

46. Bairam A, Kinkead R, Lajeunesse Y, et al. (2010) Neonatal caffeine treatment does not induce long-term consequences on TrkB receptors or BDNF expression in chemosensory organs of adult rats. Neurosci Lett 468, 292-296.

47. Xu J, Rong S, Xie B, et al. (2010) Memory impairment in cognitively impaired aged rats associated with decreased hippocampal CREB phosphorylation: reversal by procyanidins extracted from the lotus seedpod. J Gerontol $\mathbf{6 5}$, 933-940.

48. Fraga CG \& Oteiza PI (2011) Dietary flavonoids: role of (-)epicatechin and related procyanidins in cell signaling. Free Radic Biol Med 51, 813-823.

49. Terra X, Pallares V, Ardevol A, et al. (2011) Modulatory effect of grape-seed procyanidins on local and systemic inflammation in diet-induced obesity rats. $J$ Nutr Biochem 22, 380-387.

50. Narita K, Hisamoto M, Okuda T, et al. (2011) Differential neuroprotective activity of two different grape seed extracts. PloS one 6, e14575.

51. Fu W, Lu C \& Mattson MP (2002) Telomerase mediates the cell survival-promoting actions of brain-derived neurotrophic factor and secreted amyloid precursor protein in developing hippocampal neurons. J Neurosci 22, 10, 710-719.

52. Niu C (2010) The neuroprotective signaling mechanisms of telomerase via the induction by brain-derived neurotrophic factor (BDNF) in nervous system injury. Postgraduate Thesis, University of Hong Kong.

53. Wang CCLT, Liao WC, Yuan SC, et al. (2009) Cigarette smoking and cognitive impairment: a 10 year cohort study in Taiwan. Arch Gerontol Geriatr 1, 143-148. 